\title{
Programa de intervención mixta sobre el cumplimiento y calidad de vida en pacientes
} hipertensos

\author{
Luisa Fernández López* Lucia Guerrero Llamas* Josep M. Gutiérrez Villaplana ** \\ Dolors Estrada Reventos *** $\mathbf{M}^{\mathrm{a}}$ Cruz Casal Garcia* Josefina Andugar Hernández* Natividad Crespo* \\ Carlos Campo Sien* Julián Segura de la Morena en representación del Grupo EHRICA
}

*Unidad de Hipertensión. Hospital 12 de Octubre **Hospital Arnau de Vilanova. Lleida.

***Hospital Clínic. Barcelona

\section{Resumen}

El $70 \%$ de los pacientes en tratamiento antihipertensivo no controla adecuadamente sus cifras tensionales. El objetivo del estudio es comparar el impacto de diferentes intervenciones de formación de pacientes hipertensos esenciales, respecto a un grupo control sin intervención, sobre el cumplimiento terapéutico autorreferido, la presión arterial, el peso y la calidad de vida.

Ensayo clínico aleatorizado multicéntrico, con tres ramas de intervención (intervención educativa, información escrita y control) incluyendo 520 pacientes hipertensos esenciales. Las variables de estudio fueron: TA, peso, cumplimiento fármacodietético, grado de conocimiento sobre la enfermedad y calidad de vida. Completaron el estudio 428 pacientes. Analizando los resultados, los pacientes del grupo de intervención tuvieron un descenso de la PAS/PAD superior al resto de los

Correspondencia:

Luisa Fernández López

Hospital 12 de Octubre

Pabellón de Medicina Comunitaria. Unidad HTA.

Avda de Córdoba s/n

28041 Madrid

luisafernand@eresmas.com / luciaguerrero@teleline.es grupos. Así mismo, se aprecia un elevado nivel de cumplimiento farmacológico, menor cumplimiento higiénico dietético y mayor nivel de conocimientos en pacientes de centros hospitalarios. La mayoría de los pacientes, mostraron niveles muy altos de satisfacción hacia personal sanitario en los diferentes aspectos analizados.

Palabras clave:

CUMPLIMIENTO TERAPÉUTICO

HIPERTENSOS

INTERVENCIÓN EDUCATIVA

Mixed intervention programme on compliance and quality of life in hypertensive patients

\section{Abstract}

$70 \%$ of patients on treatment for hypertension do not control their blood pressure levels adequately. The aim of the study is to compare the impact of different training interventions for essential hypertensive patients, compared to a control group with no intervention, on self-referred therapeutic compliance, blood pressure, weight and quality of life. 
Multi-centre randomized clinical trail with three branches of intervention (educational intervention, written information and check-up) including 520 essential hypertensive patients. The variables in the study were BP, weight, medication and diet compliance, level of knowledge of the illness and quality of life. 428 patients completed the study. Analysing the results, patients in the group with intervention had a higher reduction in systolic/diastolic BP than the other groups. The results also showed a high level of medication compliance, lower health and diet compliance, and greater knowledge in patients in hospitals. Most of the patients showed very high levels of satisfaction towards healthcare staff in the different aspects analysed.

\section{Key words: \\ THERAPEUTIC COMPLIANCE \\ HYPERTENSIVE PATIENTS \\ EDUCATIONAL INTERVENTION}

\section{Introducción}

El $70 \%$ de los pacientes en tratamiento antihipertensivo no controla adecuadamente sus cifras de tensión arterial. La baja adherencia a las medidas terapéuticas se encuentra entre las principales causas de esta situación. Si analizamos el cumplimiento farmacológico, entre el 29 y el $56 \%$ de los pacientes hipertensos no toman la medicación de forma indicada. Esa falta de adherencia terapéutica es uno de los problemas más significativos con los que se enfrenta el personal clínico y uno de los más importantes en el control de la hipertensión.

\section{Objetivos}

Comparar el impacto de diferentes intervenciones de formación en el cumplimiento terapéutico autorreferido, la presión arterial, peso y calidad de vida en tres grupos de pacientes: control, información escrita e intervención.

\section{Material y metodos}

Estudio clínico experimental, comparativo, prospectivo y multicéntrico en una cohorte de pacientes hipertensos. La evaluación de los pacientes se realizó mediante: datos clínicos de la historia, cuestionarios de calidad de vida, conocimiento y cumplimiento al inicio y al final del proyecto.

El estudio fue realizado por enfermeras/os integrantes del Grupo EHRICA, que desarrollan su actividad en Centros de Atención Primaria y Centros de Atención Especializada y Unidades de Hipertensión de toda España. El Centro Coordinador realizó una asignación aleatoria de los centros investigadores que debían cumplir los siguientes criterios: centros de atención primaria, centros de atención especializada y unidades de hipertensión que prestaran asistencia a población hipertensa, disponer del equipo necesario para la medición de la presión arterial y disponer de una sala para las sesiones informativas.

Sujetos de estudio. El reclutamiento de los pacientes se efectuó de forma sistemática incluyendo los 30 primeros pacientes consecutivos atendidos en la consulta habitual que cumplan los siguientes criterios de selección:

\section{Criterios de inclusión:}

- Pacientes diagnosticados de hipertensión esencial leve a moderada y control aceptable de la presión arterial (sistólica $<155 \mathrm{mmHg}$ y diastólica $<95$ $\mathrm{mmHg}$ ).

- Pacientes hipertensos con independencia del tratamiento farmacológico antihipertensivo con presión arterial estable (sin modificaciones de tratamiento en los últimos 3 meses).

- Pacientes mayores de 18 años que otorgan su consentimiento escrito al estudio.

\section{Criterios de exclusión:}

- Pacientes con diagnóstico etiológico de hipertensión arterial secundaria.

- Pacientes con enfermedades sistémica importantes que precisen tratamientos y recomendaciones dietéticas específicas, como insuficiencia renal severa, insuficiencia hepática. 
- Pacientes con eventos cardiovasculares (ictus o infarto) en los últimos 3 meses.

- Pacientes con diabetes mellitus mal controlada (glucemia plasmática en ayunas $>200 \mathrm{mg} / \mathrm{dl}$ )

- Pacientes manifiestamente no cooperadores o con incapacidad para entender o cumplimentar los cuestionarios. Los pacientes se distribuyeron en tres grupos según las formación recibida con las siguientes características:

Grupo A. Programa de Intervención. Dicho programa constaba de 3 reuniones educativas semanales de 1 hora de duración:

- $1^{a}$ sesión: información sobre la hipertensión

- $2^{a}$ sesión: medidas higiénico-dietéticas

- $3^{a}$ sesión: tratamiento farmacológico

Grupo B. Programa de información escrita. Los pacientes reciben material escrito informativo al inicio del estudio, sin que se realicen sesiones educativas.

Grupo C. Programa de control. Los pacientes no recibirán ninguna intervención hasta finalizar el estudio, donde se les entrega el material escrito.

Variables de estudio. La variable principal del estudio es el nivel de presión arterial, medido en consulta clínica con la técnica habitual de esfignomanometría siguiendo las recomendaciones de la SEH-LELHA, obteniéndose el promedio de tres mediciones consecutivas.

Las variables secundarias fueron: el peso corporal, el cumplimiento farmacológico e higiénico-dietético, evaluado mediante una versión simplificada de cuestionarios validados, el grado de conocimiento de la enfermedad, según la puntuación obtenida en el "Cuestionario de conocimientos de la hipertensión", validado por Ferrán y la calidad de vida estimada con un cuestionario validado de Roca-Cusachsl.

Además se recogió información sobre las características sociodemográficas de los pacientes y su perfil clínico (antecedentes personales, factores de riesgo, hábitos y tratamiento. El estudio se desarrolló en tres visitas, y en el caso del grupo de intervención, tres sesiones adicionales, ya descritas.

Se revisaron los criterios de inclusión y exclusión en todos los pacientes hipertensos que acudieron a la consulta de enfermería, hasta completar el número de pacientes a reclutar por cada centro. Una vez que el paciente aceptó su participación se procedió con las visitas:

Visita inicial. Una vez que el paciente otorgó su consentimiento por escrito, se cumplimentaron la información de la hoja de recogida de datos y se realizaron mediciones de $\mathrm{PA}$, peso y talla, según lo descrito anteriormente. Los pacientes completaron los distintos formularios y con posterioridad se citaron de acuerdo el grupo asignado a su centro: recibir sesiones educativas, recibir material escrito 0 a formar parte del grupo control. En la visita final (3 meses) y en la visita de seguimiento (6 meses) se realizaron los mismos procedimientos que en la visita inicial.

\section{Resultados}

Centros participantes. Al inicio del proyecto participaron 54 investigadoras/es pertenecientes a centros de Atención Primaria y Unidades de HTA de toda España. Los pacientes previstos para su inclusión fueron 1620 pacientes (520 en cada grupo) y asumiendo un $20 \%$ de pérdidas, el total de pacientes previstos era de 1296 pacientes (432 por grupo).

Al final del proyecto 35 investigadores se habían retirado del proyecto, lo que supuso una pérdida de 1050 pacientes. Los 19 investigadoras/es restantes incluyeron a 570 pacientes de los que 428 completaron el estudio. Del Grupo de Información Escrita: 162 pacientes, del Grupo de Intervención: 167 y del Grupo Control 99.

Clases de centros. De los 19 investigadores, un $60 \%$ aleatorizados al grupo control pertenecían a atención primaria frente a un $40 \%$ de atención especializada. En el grupo de información escrita un 91,8\% eran de atención primaria y solo un $8,2 \%$ de atención especializada, y del grupo de intervención un $48 \%$ de atención primaria frente aun $52 \%$ a atención especializada.

Características iniciales según grupo. De los 428 pacientes reclutados, 99 lo fueron al grupo control, 162 al grupo de intervención escrita y 167 al de intervención educativa. Por sexos, eran mujeres un 
$56,3 \%$ del grupo control, un $61,7 \%$ del grupo de intervención escrita, y un 54,6\% del grupo de intervención educativa. De edades muy similares en los tres grupos, comprendidas entre los 58 a 65 años. En cuanto a la duración de la hipertensión en años, los del grupo control fueron los que más años llevaban hipertensos, una media de 12 años. En cuanto al número de antihipertensivos era similar en los tres grupos. Las cifras de presión arterial sistólica y diastólica y el índice de masa corporal al inicio fueron similares en los tres grupos, no presentando diferencias significativas entre ellos (tabla1).

\begin{tabular}{c|c|c|c|c}
\hline & CONTROL & I. ESCRITA & INTERVENCIÓN & $p$ \\
\hline N: 428 & 99 & 162 & 167 & \\
\hline Sexo (mujeres) & $56,3 \%$ & $61,7 \%$ & $54,6 \%$ & NS \\
\hline EDAD & $\mathbf{6 5 , 7} \pm 11.0$ & $65,2 \pm 8.6$ & $57,5 \pm 5.4$ & $\mathbf{0 . 0 0}$ \\
\hline $\begin{array}{c}\text { Duración HTA } \\
\text { (años) }\end{array}$ & $12,2 \pm 9.09$ & $9,3 \pm 6.2$ & $\mathbf{8 , 8} \pm 5.4$ & $\mathbf{0 . 0 0}$ \\
\hline $\begin{array}{c}\text { Antihipertensivos } \\
\text { PAS }\end{array}$ & $1,7 \pm 0.8$ & $1,6 \pm 0.9$ & $1,7 \pm 0.9$ & NS \\
\hline PAD & $82 \pm 7.2$ & $79,6 \pm 7.4$ & $\mathbf{8 3 , 9} \pm 8.7$ & $\mathbf{0 . 0 0}$ \\
\hline IMC & $29,8 \pm 4.2$ & $29,1 \pm 4.1$ & $29 \pm 4.3$ & NS \\
\hline
\end{tabular}

Tabla 1. Características iniciales de cada grupo.

Los tres grupos presentaban el mismo número de factores de riesgo cardiovascular con diferencias no significativas (tabla 2). El mayor número de pacientes diabéticos lo formaba el grupo de intervención escrita con un $26 \%$, siendo diabéticos un $11 \%$ del grupo de intervención y sólo un 7,5\% del grupo control. Un 55\% de pacientes del grupo de intervención escrita presentaban dislipemia, mientras que en los otros dos grupos la prevalencia de ésta era de un $40 \%$. El número de pacientes que presentaban lesión de órgano diana era similar en los tres grupos. El mayor número de pacientes con enfermedad cardiovascular establecida estaba entre los del grupo de intervención escrita y el grupo control con un $28,6 \%$ y un $22,5 \%$ respectivamente, mientras que los del grupo de intervención educativa sólo presentaban enfermedad cardiovascular un $9.2 \%$. En cuanto a la situación laboral un $40 \%$ de los pacientes del grupo de intervención educativa estaban en activo. El nivel educacional era en general bajo, los pacientes del grupo de información escrita eran los que presentaban mayor nivel educacional con un $20,4 \%$ (tabla 3 ).

\begin{tabular}{|c|c|c|c|c|}
\hline & CONTROL & I. ESCRITA & INTERVENCIÓN & $\mathbf{p}$ \\
\hline$N: 428$ & 99 & 162 & 167 & \\
\hline $\mathrm{N}^{\circ}$ factores RCV & $1,3 \pm 1.1$ & $1,5 \pm 0.9$ & $1,35 \pm 0.9$ & 0.07 \\
\hline Diabetes mellitus & $7.5 \%$ & $26 \%$ & $11.2 \%$ & 0.00 \\
\hline Dislipemia & $40 \%$ & $55.1 \%$ & $40.8 \%$ & 0.011 \\
\hline $\begin{array}{l}\text { Lesión órgano } \\
\text { diana }\end{array}$ & $17.5 \%$ & $12.2 \%$ & $12.5 \%$ & 0.47 \\
\hline Enf. cardiovascular & $22.5 \%$ & $28.6 \%$ & $9.2 \%$ & 0.00 \\
\hline
\end{tabular}

Tabla 2. Riesgo cardiovascular de los grupos.

\begin{tabular}{c|c|c|c}
\hline & CONTROL & I. ESCRITA & INTERVENCIÓN \\
\hline $\mathrm{N}: 428$ & 99 & 162 & 167 \\
\hline $\begin{array}{c}\text { Situación laboral } \\
\text { (Activo) }\end{array}$ & $15 \%$ & 16.8 & 40.1 \\
\hline $\begin{array}{c}\text { Nivel educacional } \\
\text { (leer y escribir) }\end{array}$ & $6.3 \%$ & $20.4 \%$ & $7.9 \%$ \\
\hline
\end{tabular}

Tabla 3. Situación laboral y nivel educacional.

Reducción de la Presión Arterial (figura 1). Con respecto a las cifras de presión arterial comparando visita de inicio con final en los diferentes grupos podemos ver que los pacientes del grupo de intervención tuvieron un descenso de la PAS/PAD superior al resto de los grupos. El cambio de la presión arterial sistólica depende de la presión sistólica al inicio, del grupo de asignación y de la edad del paciente.

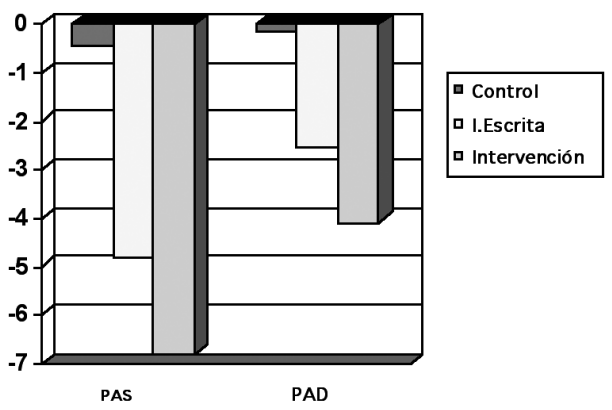

Figura 1. Reducción de la Presión Arterial.

Test de conocimiento (figuras 2 y 3 ). Tras realizar un análisis de varianza, aparecen diferencias significativas en el grupo de intervención frente a los otros grupos, únicamente en la variable conocimiento. En 
el resto de las variables analizadas, comentadas anteriormente, no se observaron efectos significativos.

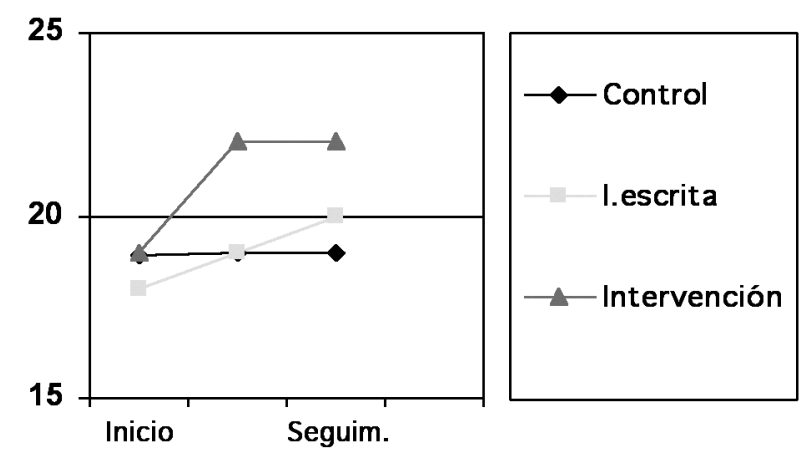

Figura 2. Test de conocimientos.

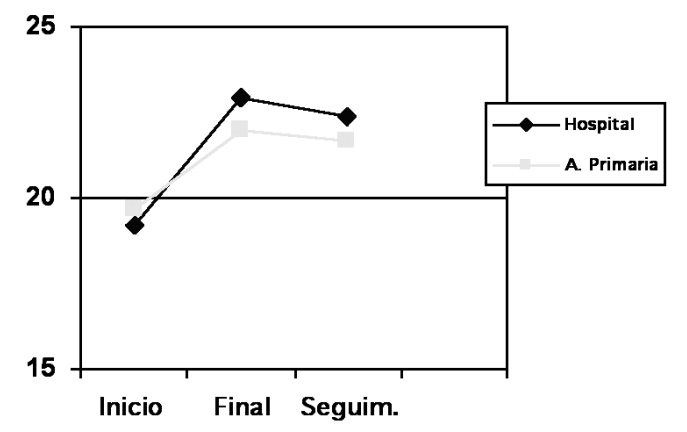

Figura 3. Grado de conocimiento según centro.

Se analizaron si las características previas de los sujetos como: sexo, edad, estudios, clase social etc, tenían relación con la eficacia del programa. Sólo se han encontrado diferencias significativas en el aumento del nivel de conocimiento de los pacientes de centro hospitalario frente a los de atención primaria

Cumplimiento farmacológico (figura 4). Podemos señalar que los pacientes incluidos en este estudio manifiestan un elevado nivel de cumplimiento farmacológico auto informado, donde más del $97 \%$ de los sujetos consumen entre el $80 \%$ y $100 \%$ de la medicación prescrita.

Cumplimiento higienico-dietético (tabla 4). Los niveles de cumplimiento higiénico-dietético autoinformados son significativamente inferiores a los alcanzados en el tratamiento farmacológico. En este sentido, el seguimiento de la dieta hipocalórica obtendría los peores resultados (solo un $40,9 \%$ de los sujetos la siguen diariamente), mientras que únicamente el $57 \%$ informan realizar ejercicio físico a diario, y por último casi un $65 \%$ de los pacientes señalan realizar una dieta hiposódica de modo regular.

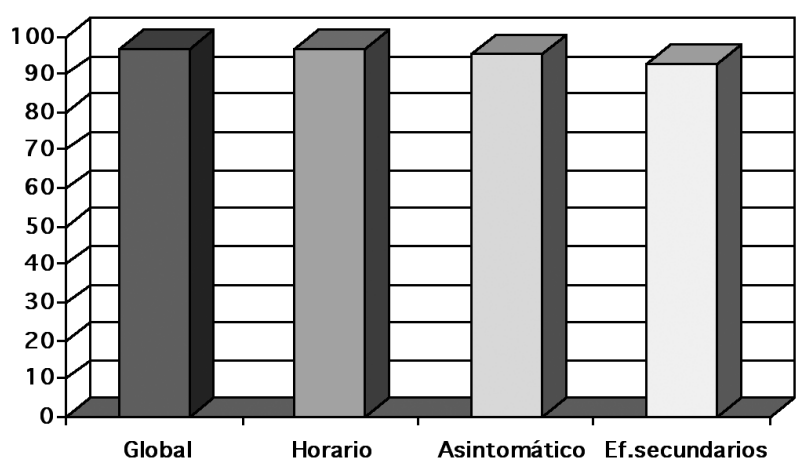

Figura 4. Cumplimiento farmacológico.

\begin{tabular}{c|c|c|c}
\hline Frecuencia & Dieta lípośdica & Dieta lipocalóric a & $\begin{array}{c}\text { Práctica ejercic io } \\
\text { fisico }\end{array}$ \\
\hline Nunca & $13,5 \%$ & $22,1 \%$ & $9,9 \%$ \\
\hline $\begin{array}{c}\text { 1 a 2 díns por } \\
\text { semana }\end{array}$ & $10,1 \%$ & $15,4 \%$ & $16,1 \%$ \\
$\begin{array}{c}\text { 3 a 4 díns por } \\
\text { semana }\end{array}$ & $11,3 \%$ & $21,3 \%$ & $16,1 \%$ \\
\hline \begin{tabular}{c} 
Dinriamente \\
\hline
\end{tabular} & $64,8 \%$ & $40,9 \%$ & $57,3 \%$ \\
\hline
\end{tabular}

Tabla 4. Cumplimiento higiénico-dietético.

En cuanto a las variables psicológicas analizadas cabe señalar:

Expectativas de autoeficacia (figura 5). En general los pacientes se sienten muy capaces de llevar el tratamiento farmacológico; por el contrario, el seguimiento de la dieta hipocalórica representa el área en la que los pacientes se sienten menos capaces de llevarlo a cabo

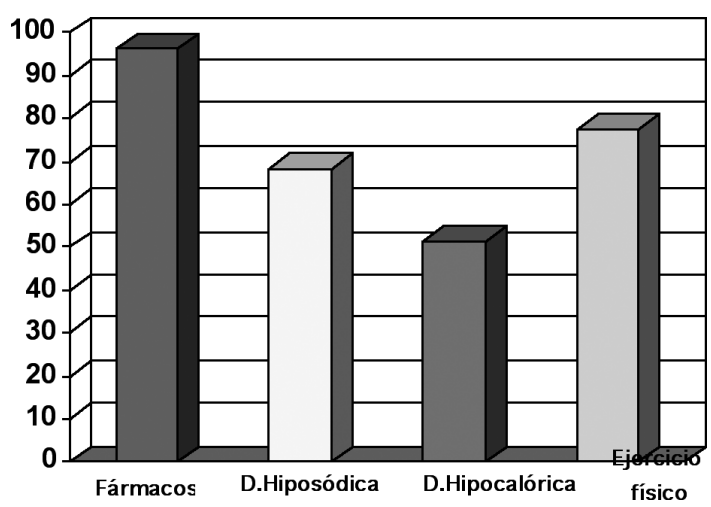

Figura 5. Expectativa de autoeficacia. 
Costo percibido (figura 6). En este gráfico podemos ver que el tratamiento farmacológico les supone un bajo nivel de costos, en cambio el seguimiento de la dieta hipocalórica les supone un alto nivel de costos

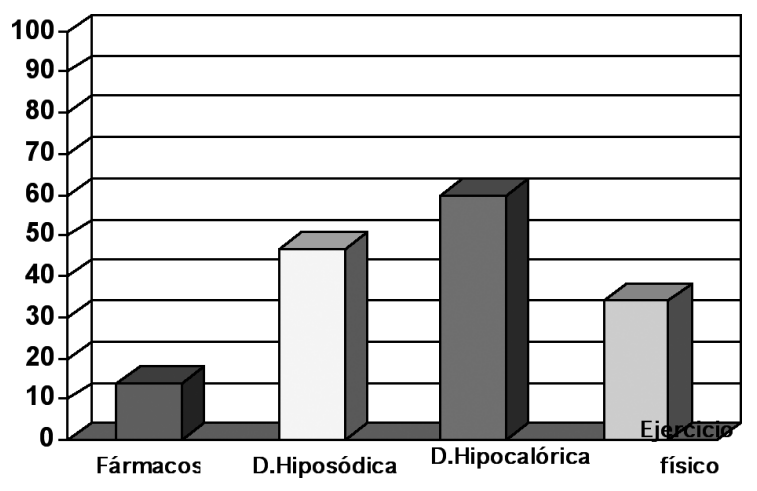

Figura 6. Costo percibido.

Apoyo social (figura 7). En relación con el apoyo social, los resultados demuestran que algo más de la mitad de los sujetos señalan tener poco apoyo en el seguimiento de las diferentes dimensiones del tratamiento para la hipertensión.

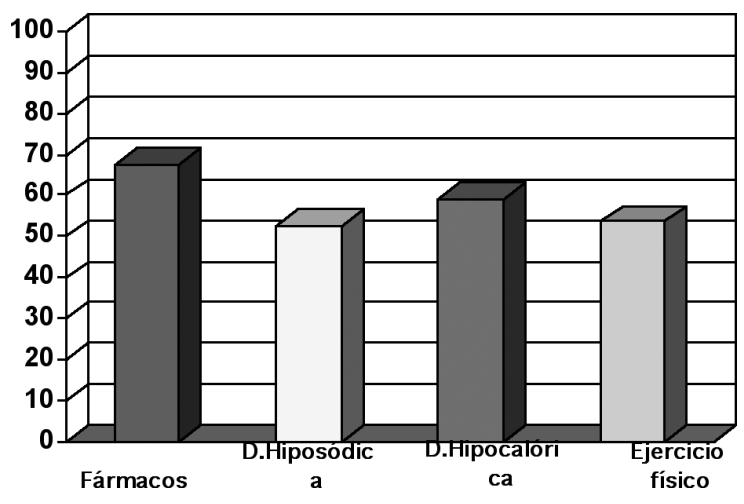

Figura 7. Apoyo social percibido.

\section{Conclusiones}

Ni en el grupo control ni en el que recibió información escrita hay cambios significativos. En el grupo de intervención no hay cambios significativos en la calidad de vida y en el cumplimiento farmacológico e higiénico-dietético, pero si hay cambios en la variable conocimiento, con diferencias por centro de atención. Cabe destacar el elevado nivel de cumplimiento farmacológico, el bajo nivel de cumplimiento higiénicodietético, el elevado nivel de satisfacción hacia el personal sanitario y que la duración de la consulta es suficiente y el tiempo de espera normal. Por tanto, se puede afirmar que la aplicación del grupo de intervención se ha mostrado efectiva para mejorar el nivel de presión arterial y de conocimiento frente a los grupos control e información escrita y que los grupos educativos dirigidos por la enfermería mejoran el conocimiento del paciente sobre su proceso y mejora el control de presión arterial, lo que contribuye a mejorar el perfil de riesgo de nuestra población.

\section{Bibliografía}

1. A Coca. Evolución de la hipertensión arterial en España. Resultados del Estudio Controlpres 2001. Hipertensión 2002; 19(9):390-399.

2. Torre J, Fernández R, López J, Nicolás M, López F, Pérez F. Estudio del cumplimiento del tratamiento y control HTA en un medio urbano. Rev Clin Esp 1990; 187: 103-106.

3. Gil V, Belda J, Muñoz C, Martínez VJ, Muñoz C, Soriano JE, Merino J. Validez de cuatro métodos indirectos que valoran el cumplimiento terapéutico en la hipertensión arterial. Rev Clin Esp 1993; 193: 363-367.

4. Guía sobre el diagnóstico y el tratamiento de la hipertensión arterial en España 2002. Sociedad Española de Hipertensión Liga Española para la lucha contra la Hipertensión Arterial (SEH-LELHA). Hipertensión 2002; 19 (Supl 3).

5. Joint National Committee: The Seventh Report of the Joint National Committee on Detection, Evaluation, and Treatment of High Blood Pressure. JAMA 2003; 289: 2560-2572.

6. 1999 World Health Organitation-International Society of Hypertension Guidelines for the Manegement of Hypertensión J. Hypertens. 1999, 17: 151-185.

7. Kyngäs H, Lahdenperä T. Compliance of patients with hypertension and associated factors. Journal of Advanced Nursing, 1999; 29: 832-839.

8. Martínez Amenós A. La observancia en el tratamiento de la hipertensión arterial. Barcelona: Ed. Doyma, 1993.

9. Ferrer V. "Adherencia" o "cumplimiento" de prescripciones terapéuticas y de salud: concepto y factores psicosociales implicados. Revista de Psicología de la Salud 1995; 7(1): 35-59. 
10. Meichenbaum D, Turk DC. Faciliting Treatment Adherence. A practitioner's Guidebook. New York: Plenum Press, 1987.

11. N. Crespo, VJ Rubio, MI Casado, J Sánchez, C. Campo. Factores moduladores de la adherencia farmacológica en hipertensión arterial: estudio exploratorio. Hipertension 2000; 17:4-8

12. Ferrán $M$, Parcet J, Casabella $B$, Fernández $M$, de la Torre M. Educación sanitaria a pacientes hipertensos. Propuesta de un test de conocimiento y cumplimiento. Aten Primaria 1988; 5: 2531.

13. Roca-Cusachs A, Badia D, Antoni.Gascón, G, AbeIlán J, Lahoz R, Varela C, Velasco, 0. Relación entre variables clínicas y terapéuticas y calidad de vida relacionada con la salud en pacientes con hipertensión arterial. Estudio MINICHAL. Med Clín (Barc) 2003; 121:12-17. 